\title{
MAGNETIC FILAMENTS IN THE NEGATIVE-LATITUDE EXTENSION OF THE RADIO ARC NEAR THE GALACTIC CENTER
}

\author{
F. YUSEF-ZADEH \\ Dept. of Physics and Astronomy, Northwestern University \\ Evanston, IL 60201
}

MARK MORRIS

Dept of Astronomy, UCLA, Los Angeles, CA 90024

\author{
A.N. LASENBY \\ MRAO, Cavendish Laboratory, Madingley Road \\ Cambridge, CB3 0HE, UK
}

\section{J.H. SEIRADAKIS \\ University of Thessaloniki, Dept. of Physics \\ GR-54006, Thessaloniki, Greece}

\section{R. WIELEBINSKI \\ Max-Planck-Institut für Radioastronomie Auf dem Hügel 69, D5300 Bonn 1, W. Germany}

\begin{abstract}
Continuum observations of the southern extension of the radio Arc located near $1 \sim 0.2^{\circ}$ have been carried out at $\lambda 20$ and $6 \mathrm{~cm}$ using the VLA in its hybrid $B / C$ and $C / D$ array configurations. $A$ number of long and narrow filaments have been identified on the negative latitude side of the plane. Some of the filaments appear to extend continuously into the radio continuum Arc and suggesting strongly that they are associated physically with the Arc. Other filaments appear isolated and thus have characteristics similar to those of the radio "threads" which have been seen near the Galactic center. These new threads and filaments are highly polarized at $\lambda 6 \mathrm{~cm}$ and show rotation measures which vary between 300 and $3000 \mathrm{rad} \mathrm{m}^{-2}$. The details present in the highresolution images of this region strengthen the hypotheses that the large field strength is dynamically important and that the large-scale geometry of the magnetic field is poloidal near the Galactic center.
\end{abstract}

Radio continuum observations of the inner degree of the galactic center region using single-dish telescopes have revealed three polarized sources at $\lambda 3 \mathrm{~cm}$ : a relatively compact one located along the Arc and identified as G0.16-0.15, while other more extended structures are situated along the northwest and southeast extenstions of the Arc on opposite sides of the galatic plane. In earlier high-resolution radio continuum observations, the filamentary structures of the Arc and its northeast extensions were shown to be physically associated with each other. Here we present high-resolution radio images of the region to the negative-latitude side of the radio Arc. The results of these observations will be given elsewhere. 


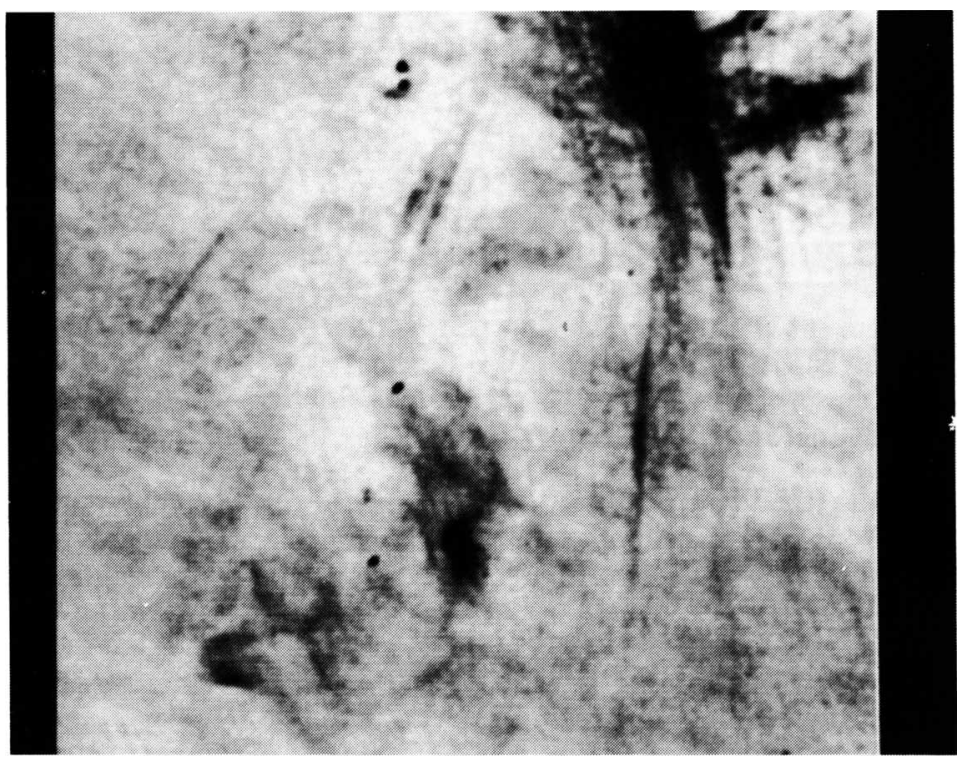

Fig. 1 [top]: The $\lambda 20 \mathrm{~cm}$ total intensity image of the negative-latitude side of the galactic plane in $1 \times b$ where a number of new filaments can be identified. The bright extended structures to the northwest is associated with the radio Arc.

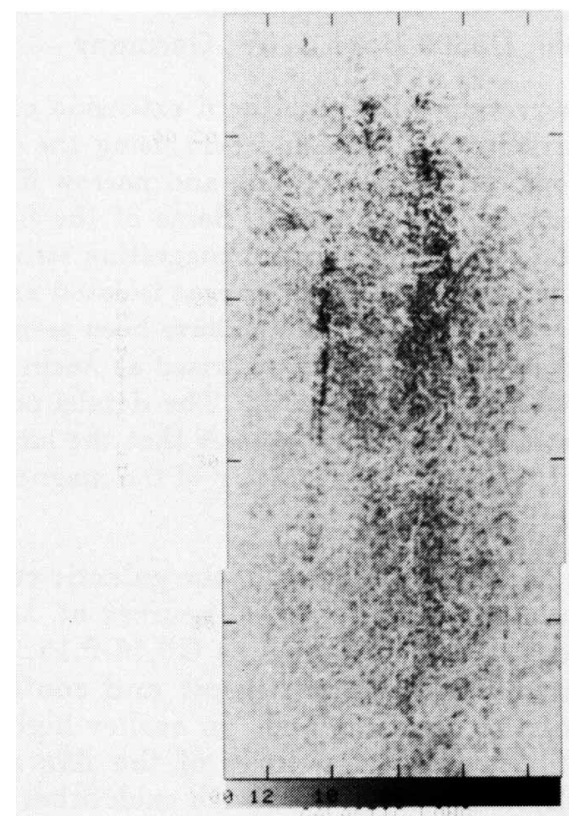

Fig. 2 /bottom]: The polarized intensity image of most prominent of the narrow filaments shown in Fig. 1. This figure is constructed by mosaicking three adjacent $\lambda 6 \mathrm{~cm}$ fields in order to cover the $20^{\prime}$ extent of this filament in the direction perpendicular to the galactic plane. 